\title{
Relações entre características de crescimento no curimbatá (Prochilodus lineatus), considerando as famílias de meio-irmãos maternos
}

\author{
Relationship among growth characteristics in curimbatá \\ (Prochilodus lineatus), considering halfsib families
}

\author{
Marco Antonio da Rocha, ${ }^{*}$ Edson Luis de Azambuja Ribeiro, ${ }^{*}$ Ivone Yurika Mizubuti, ${ }^{*}$ \\ Leandro das Dores Ferreira da Silva*
}

\begin{abstract}
Resumo
Em uma amostra constituída de cinco famílias de meio-irmãos, descendentes de cinco fêmeas e dez machos (acasalamento hierárquico), com 20 indivíduos por família, foram determinadas a taxa de crescimento médio (igr) e as relações entre as características: peso corporal, comprimento corporal, altura corporal e comprimento da cabeça no curimbatá (Prochilodus lineatus). A igr calculada entre as idades conforme as famílias de meio-irmãos mostrara uma certa similaridade, e as relações entre as características mostraram, de uma forma geral, um crescimento alométrico com exceção para a relação altura corporal e comprimento corporal.
\end{abstract}

Palavras-chave: peixes; biometria; aquicultura; alometria; isometria.

\begin{abstract}
Data came from a sample of 5 curimbata (P. lineatus) halfsib families, out of 5 dams and 10 sires (nested mating). twenty fishes per family were used to determine the instant growth rate (igr) and the relationship among body weight, body length, body high and head length. The calculated igr between ages, according to halfsib families, showed a certain similarity, and the relationships between traits showed an alometric growth, the only exception was the relationship between body high and body length.
\end{abstract}

Keywords: fishes; biometry; aquaculture; alometric; isometric.

\section{Introdução}

O crescimento dos peixes pode ser medido através do peso, do comprimento corporal em função da idade, e outros. De acordo com Royce (1972), o crescimento pode ter uma influência endógena representada pela herança. Os fatores exógenos são representados por um complexo de fatores ambientais, sendo provavelmente a quantidade e a qualidade dos alimentos o mais importante.

A taxa de crescimento para peixes entre intervalos de tempo pode ser calculada pela taxa de crescimento instantânea (igr). As diferentes partes do corpo dos peixes podem ter taxas de crescimento similares (crescimento isométrico) quando o coeficiente de regressão (b) é igual a 1,0, isto é, as duas partes do corpo crescem de forma proporcional. Por outro lado, um valor de $b>1$ indica que uma das partes desenvolve mais que a outra, sendo chamada de alométrica.

Estas relações no Curimbatá, conforme Leite et al. (1984), apresentaram entre altura e comprimento, um crescimento que pode ser considerado como isométrico $(b=1,06)$, e a relação comprimento da cabeça e comprimento corporal total $(b=0,82)$ que pode se considerado como alométrico.
A relação entre o peso e o comprimento corporal total pode ter um crescimento isométrico quando $b=3,0$, isto é, o peso aumenta com o comprimento corporal elevado ao cubo. No entanto, quando $b<3$, o incremento é maior no comprimento. Por outro lado, quando $b>3$, o maior desenvolvimento é relativo ao peso.

Os valores de b na relação peso e comprimento corporal para peixes situam-se entre 2,5 e 4,0 (Le Cren, 1951) ou 2,0 e 3,5 (Royce, 1972). Geralmente os valores de b giram em torno de 3,0 (crescimento isométrico), em truta-arco-íris (Gunnes e Gjedrem, 1981; Gjerde e Schaffer, 1989) e no salmão (Refstie e Steine 1978). No curimbatá os valores de b são também próximos de 3,0, conforme Verani et al. (1989) com 3,26, Leite et al. (1984) com o valor de 3,02, Mainardes Pinto et al. (1984) com $b=2,95$ e Toledo Filho e Santos (1987) citados em GaIhardo (1989) com $b=3,00$.

O objetivo deste trabalho foi determinar a taxa de crescimento e as relações entre as características: peso corporal, comprimento corporal total, altura corporal e comprimento da cabeça em famílias de meio-irmãos maternos, no Curimbatá (Prochilodus lineatus). 


\section{Material e métodos}

O experimento foi realizado na estação de piscicultura da Universidade Estadual de Londrina. As fêmeas e os machos utilizados na reprodução tiveram três origens: piscicultura particular, capturados na natureza, e de uma estação de piscicultura pertencente ao IAP, órgão do Governo do Paraná.

Utilizou-se um acasalamento hierárquico, com cinco fêmeas e dez machos, sendo os machos dentro de fêmeas. A desova foi feita por indução com hipófise de carpa. Após a fecundação e a hidratação dos ovos, estes foram transferidos para as incubadoras. Nas incubadoras a água foi constantemente renovada, à temperatura de aproximadamente $28^{\circ} \mathrm{C}$, e com fonte de ar comprimido.

Após um período de aproximadamente 24 horas, as larvas eclodiram e, durante os primeiros quatro dias, elas se alimentaram do conteúdo do saco vitelínico. Após o quarto dia de vida, as larvas começaram a alimentação por via oral. No período em que elas ficaram nas incubadoras (dez dias após o nascimento), foram alimentadas com ovo de galinha floculado, na quantidade de $20 \mathrm{ml}$ de ovo floculado/incubadora, três vezes ao dia.

Após o décimo dia do nascimento, as larvas foram transferidas para um viveiro externo, de $100 \mathrm{~m}^{2}$ de superfície e $1,2 \mathrm{~m}$ de profundidade. Como estes animais não permitiam qualquer tipo de marcação, eles foram transferidos para tanques-rede com dimensões de $2,25 \mathrm{~m}^{2}$ de superfície e altura de $1,7 \mathrm{~m}$. Um total de dez tanques-rede foi instalado com fonte de ar comprimido, sendo que cada tanque representava uma família de irmãos inteiros.

Além da alimentação natural, presente nos viveiros, iniciouse a alimentação artificial com uma ração contendo $40 \%$ de proteína bruta e $2.900 \mathrm{kcal}$ de energia metabolizável. Da fase de alevinagem até o final do experimento os animais receberam uma ração peletizada com $30 \% \mathrm{~Pb}$ e $2.800 \mathrm{kcal}$ de energia metabolizável na quantidade de $3 \%$ da biomassa.

Após a idade de 150 dias, os animais foram marcados para a identificação das famílias de meio-irmãos maternos pelo método de Welsh e Mills (1981). Em seguida, foram misturados e colocados em cinco viveiros de aproximadamente $100 \mathrm{~m}^{2}$ de superfície e $1,2 \mathrm{~m}$ de profundidade.

Após a transferência dos animais para os viveiros, algumas características da água foram determinadas, com o objetivo de comparação entre as condições desses parâmetros determinados no reservatório de abastecimento e nos viveiros. Desta forma, a cada 15 dias, e no mesmo horário, foram determinados a temperatura, $\mathrm{pH}$, condutividade elétrica e 0 oxigênio dissolvido.

As características utilizadas para determinar as relações foram: peso corporal, comprimento corporal total, altura e comprimento da cabeça, nas idades de 60, 90, 150, 210, 270 e 330 dias.

As relações entre peso e altura, altura e comprimento corporal total, comprimento da cabeça e comprimento corporal total, peso e comprimento corporal total foram determinados com os valores abrangendo o período de 60 até 330 dias.

Inicialmente as variáveis foram transformadas para logaritmo natural. Em seguida, foram determinados o A (intercepto) e o $B$ (coeficiente de regressão). $O$ intercepto calculado através da transformação não representa a constante da relação exponencial. $O$ cálculo é feito da seguinte forma: $\ln a=e^{-A}$

em que:

Ina = intercepto Ina

$\mathrm{e}=$ antilogaritmo natural

$A=$ intercepto calculado com a transformação das variáveis para logaritmo natural.

O teste para isometria ou alometria ao nível de significância de $5 \%$ foi feito através do teste " $t$ ", conforme Sokal e Rohlf (1980).

As características avaliadas da qualidade da água foram analisadas conforme o modelo:

$$
Y_{i j k}=\mu+V_{1}+M_{1}+(V M)_{l j}+E_{i j k}
$$

Em que:

$\mathrm{Y}_{\mathrm{ijk}}=$ temperatura, $\mathrm{pH}$, condutividade elétrica e oxigênio dissolvido da K-ésima determinação no i-ésimo viveiro, no jésimo mês.

$\mu=$ média geral;

$V_{i}=$ efeito do i-ésimo viveiro;

$M_{j}=$ efeito do j-ésimo mês;

$\mathrm{VM}=$ efeito da interação entre o i-ésimo viveiro com o j-ésimo mês:

$E_{i j k}=$ erro experimental

Para as comparações de médias foi utilizado o teste de Tukey ao nivel de $5 \%$ de probabilidade.

A taxa de crescimento instantâneo (igr) entre as médias dos pesos corporais nas idades de 60, 90, 150, 210, 270 e 330 dias para grupos de meio-irmãos foi determinada pela fórmula apresentado por Ricker (1979):

$$
\text { igr }=\left(\ln W_{f}-\ln W_{t}\right) /\left(t_{f}-t_{f}\right)
$$

Em que:

In $\mathrm{W}_{\mathrm{f}}=$ logaritmo natural do peso na idade final

In $W_{i}=$ logaritmo natural do peso na idade inicial

$t_{f}=$ idade final

$t_{i}=$ idade inicial

\section{Resultados e discussão}

Na Tabela 1 são apresentados as médias, desvio-padrão residual e coeficiente de variação para as características avaliadas na água, conforme os meses.

A temperatura, $\mathrm{pH}$ e condutividade elétrica não apresentaram efeito significativo $(P>0,05)$ entre viveiros e para a interação viveiros $x$ meses. No entanto, apresentavam efeito significativo entre meses $(P<0,01)$.

O oxigênio dissolvido ( $\mathrm{mg} /$ litro) apresentou diferenças significativas $(P<0,01)$ entre viveiros e entre meses, não apresentando efeito significativo para a interação viveiros $x$ meses ( $P>0,05)$.

$\mathrm{Na}$ Tabela 2, são apresentados as médias, desvios-padrão e coeficientes de variação para a característica oxigênio dissolvido na água, conforme os viveiros. 
Tabela 1 - Médias, desvio-padrão residual e coeficiente de variação para as características avaliadas na água, conforme os meses

\begin{tabular}{lcccc}
\hline Meses & $\begin{array}{r}\text { Temperatura } \\
\text { da água }\left({ }^{\circ} \mathrm{C}\right)\end{array}$ & pH & $\begin{array}{c}\text { Condutividad } \\
\text { e Elétrica } \\
\left(\mu \mathbf{S . C m} \mathbf{-}^{-}\right)\end{array}$ & $\begin{array}{c}\text { Oxigênio } \\
\text { dissolvido } \\
(\mathbf{m g} / \mathbf{l})\end{array}$ \\
\hline Agosto & $18,25 \mathrm{c}$ & $6,86 \mathrm{~b}, \mathrm{c}$ & $55,05 \mathrm{~b}$ & $7,50 \mathrm{a}$ \\
Setembro & $21,58 \mathrm{~b}$ & $6,69 \mathrm{c}$ & $58,26 \mathrm{a}, \mathrm{b}$ & $6,89 \mathrm{~b}$ \\
Outubro & $24,08 \mathrm{a}$ & $7,41 \mathrm{a}, \mathrm{b}$ & $64,46 \mathrm{a}$ & $6,61 \mathrm{~b}$ \\
Novembro & $23,50 \mathrm{a}, \mathrm{b}$ & $7,56 \mathrm{a}$ & $54,46 \mathrm{~b}$ & $6,62 \mathrm{~b}$ \\
Dezembro & $24,00 \mathrm{a}$ & $6,84 \mathrm{~b}, \mathrm{c}$ & $54,62 \mathrm{~b}$ & - \\
Janeiro & $23,33 \mathrm{~b}$ & $6,58 \mathrm{c}$ & $55,08 \mathrm{~b}$ & \\
\hline Desvio-padrão & 1,82 & 0,58 & 5,18 & 0,49 \\
Residual & 8,10 & 8,30 & 9,09 & 7,10 \\
CV (\%) & & & & \\
\hline
\end{tabular}

Nas colunas, médias seguidas de letras iguais não diferem estatisticamente pelo teste de Tukey $(P>0,05)$

Tabela 2 - Médias (X), desvios-padrão(s) e coeficientes de variação (CV) para a característica oxigênio dissolvido na água, conforme os viveiros

\begin{tabular}{cccc}
\hline Viveiros & $\begin{array}{c}\mathbf{x} \\
(\mathbf{m g} / \mathbf{l})\end{array}$ & $\begin{array}{c}\mathbf{S} \\
(\mathbf{m g} / \mathbf{l})\end{array}$ & $\begin{array}{c}\mathbf{C . V} \\
(\mathbf{\%})\end{array}$ \\
\hline 1 & $7,82 \mathrm{a}$ & 0,65 & 8,30 \\
2 & $6,75 \mathrm{~b}$ & 0,62 & 9,20 \\
3 & $6,86 \mathrm{~b}$ & 0,64 & 9,31 \\
4 & $6,52 \mathrm{~b}$ & 0,65 & 9,92 \\
5 & $6,64 \mathrm{~b}$ & 0,47 & 7,11 \\
6 & $6,82 \mathrm{~b}$ & 0,64 & 9,39 \\
\hline
\end{tabular}

Médias seguidas de letras iguais não diferem estatisticamente pelo teste de Tukey $(P>0,05)$.

Foi observada diferença significativa $(P<0,05)$ do viveiro 1 em relação aos outros viveiros.

$\mathrm{Na}$ Tabela 3 são apresentados os valores do intercepto, coeficiente de regressão, o coeficiente de determinação e o tipo de crescimento para a relação peso corporal e altura, segundo as famílias. Na Tabela 4, para a relação altura corporal e comprimento corporal total; na Tabela 5, para a relação comprimento da cabeça e comprimento corporal total e, na Tabela 6, para a relação peso corporal e comprimento total.

O valor das constantes na Tabela 3, calculado com a amostra total, foi muito próximo ao valor encontrado por Leite et al. (1984). Em relação às famílias de meio-irmãos maternos verifica-se que esta relação, representada pelo coeficiente de regressão (b), mostra que o crescimento é alométrico negativo $(P<0,05)$, isto é, a característica peso corporal tem crescimento proporcionalmente maior que a característica altura corporal.

A Tabela 4 mostra um crescimento isométrico, ou seja, com valor de $b=1,00$. Este valor encontrado é muito semelhante ao determinado por Leite et al. (1984). Isso representa um crescimento uniforme da altura corporal e comprimento corporal total. No entanto, a alometria positiva foi encontrada na relação entre as duas variáveis para o grupo de meio-irmãos da fêmea nº 2 .

$\mathrm{Na}$ Tabela 5 o valor calculado na amostra total é inferior aos valores encontrados por LEITE et al (1984). Os valores do coeficiente de regressão (b) indicam crescimento alométrico negativo $(P>0,05)$, isto é, o comprimento corporal total apresentou crescimento proporcional maior que o crescimento do comprimento da cabeça em todas as famílias.
Tabela 3 - Valores do intercepto (Ina); coeficiente de regressão (b) e coeficiente da determinação $\left(r^{2}\right)$ e tipo de crescimento (cresc.), para a relação peso corporal e altura, segundo as famílias

\begin{tabular}{lcccc}
\hline Famílias & Ina & $\mathbf{b}$ & $\mathbf{r}^{\mathbf{2}}$ & cresc. \\
\hline Fêmea 1 & 0,6844 & 2,93 & 0,99 & Alométrico \\
Fêmea 2 & 0,7870 & 2,87 & 0,99 & Alométrico \\
Fêmea 3 & 0,7165 & 2,88 & 0,99 & Alométrico \\
Fêmea 4 & 0,7280 & 2,90 & 0,99 & Alométrico \\
Fêmea 5 & 0,7339 & 2,88 & 0,99 & Alométrico \\
\hline Geral & 0,7226 & 2,89 & 0,99 & Alométrico \\
\hline
\end{tabular}

Alométrico $(P<0,05)$, pelo teste " $t$ ".

Tabela 4 - Valores do intercepto (Ina), coeficiente de regressão (b), coeficiente de determinação $\left(r^{2}\right)$ e tipo de crescimento (cresc.), para a relação altura corporal e comprimento corporal, segundo as famílias

\begin{tabular}{lcccc}
\hline Famílias & Ina & $\mathbf{b}$ & $\mathbf{r}^{2}$ & cresc. \\
\hline Fêmea 1 & 0,2838 & 0,99 & 0,98 & Isométrico \\
Fêmea 2 & 0,2560 & 1,02 & 0,99 & Alométrico \\
Fêmea 3 & 0,2771 & 1,01 & 0,98 & Isométrico \\
Fêmea 4 & 0,2661 & 1,01 & 0,98 & Isométrico \\
Fêmea 5 & 0,2760 & 1,01 & 0,98 & Isométrico \\
\hline Geral & 0,2743 & 1,00 & 0,98 & Isométrico \\
\hline
\end{tabular}

Alométrico $(P<0,05)$, isométrico $(P>0,05)$, pelo teste "t".

Tabela 5 - Valores do intercepto (Ina), coeficiente de regressão (b), coeficiente de determinação $\left(r^{2}\right)$ e tipo de crescimento (cresc.), para a relação comprimento da cabeça e comprimento corporal, segundo as famílias.

\begin{tabular}{lcccc}
\hline Famílias & Ina & $\mathbf{b}$ & $\mathbf{r}^{2}$ & cresc. \\
\hline Fêmea 1 & 0,4087 & 0,78 & 0,97 & Alométrico \\
Fêmea 2 & 0,4051 & 0,78 & 0,98 & Alométrico \\
Fêmea 3 & 0,4076 & 0,78 & 0,97 & Alométrico \\
Fêmea 4 & 0,4139 & 0,77 & 0,95 & Alométrico \\
Fêmea 5 & 0,3931 & 0,78 & 0,96 & Alométrico \\
\hline Geral & 0,4065 & 0,78 & 0,97 & Alométrico \\
\hline
\end{tabular}

Alométrico $(P<0,05)$, pelo teste " $t$ ".

Tabela 6 - Valores do intercepto (Ina), coeficiente de regressão (b), coeficiente de determinação $\left(r^{2}\right)$ e tipo de crescimento (cresc.), para a relação peso corporal e comprimento total, segundo as famílias

\begin{tabular}{lcccc}
\hline Famílias & Ina & $\mathbf{b}$ & $\mathbf{r}^{\mathbf{2}}$ & cresc. \\
\hline Fêmea 1 & 0,01582 & 2,93 & 0,99 & Alométrico \\
Fêmea 2 & 0,01458 & 2,97 & 0,99 & Isométrico \\
Fêmea 3 & 0,01661 & 2,93 & 0,99 & Alométrico \\
Fêmea 4 & 0,1454 & 2,96 & 0,99 & Alométrico \\
Fêmea 5 & 0,01639 & 2,94 & 0,99 & Alométrico \\
\hline Geral & 0,01575 & 2,94 & 0,99 & Alométrico \\
\hline
\end{tabular}

Alométrico $(P<0,05)$, isométrico $(P>0,05)$, pelo teste " $t$ ". 
O valor apresentado na Tabela 6, considerando a amostra total, indicou um valor de $b=2,94$, o que sugere ser esta relação alométrica negativa $(p<0,05)$ e dentro da faixa de valores citados em Le Cren (1951) e Royce (1972). Comparando este valor com o encontrado no Curimbatá por Mainardes Pinto et al. (1984), verifica-se que é praticamente o mesmo valor de b. Por outro lado, é um valor muito próximo aos encontrados por Gunnes e Gjedrem (1981), Gjerde e Schaffer (1989) na truta-arco-íris, Refstie e Steine (1978) no Salmão, e Leite et al. (1984) e Toledo Filho e Santos (1987) citado em Galhardo (1989) e inferior ao valor determinado por Verani et al. (1989) no Curimbatá. Observa-se também nesta tabela, que o valor do coeficiente de regressão (b) e o intercepto (Ina) estão associados negativamente, isto é, valores maiores de $b$ indicam valores menores de Ina e vice-versa. Esta observação está de acordo com Le Cren (1951). Nesta relação, todas as famílias de meio-irmãos maternos oriundos das fêmeas 1,3 e 5 apresentaram alometria negativa, com exceção da fêmea 2.

$\mathrm{Na}$ Tabela 7, são apresentadas as taxas de crescimento instantâneo (igr), conforme a idade, segundo as fêmeas.

$O$ incremento observado nesta tabela foi em relação ao igr entre as idades de 60 a 90 dias, em todas as famílias. Entre as idades de 90 a 150 dias, houve uma redução do igr em todas as famílias, e pode ter sido decorrência do tamanho dos animais e o espaço disponivel (tanque-rede). Entre as idades de 150 e 210 dias, o igr aumentou em todos os grupos em função da transferência dos animais dos tanquesrede para os viveiros. Em idades posteriores o crescimento não foi acentuado.

\section{Conclusões}

O crescimento uniforme (isométrico) foi observado na relação altura corporal e comprimento corporal para todas as famílias, à exceção da fêmea $n^{\circ} 2$, que apresentou crescimento alométrico. As outras relações apresentaram crescimento alométrico para todas as famílias.

\section{Referências}

GALHARDO, E. Estudo genético do polimorfismo de transferrinas em populações selvagens e cultivadas de curimbatá, Prochilodus Scrofa (Peixes, Prochilodontide). $123 \mathrm{f}$. Dissertação (Mestrado) - Departamento de Biologia. Instituto de Biociências, Universidade de São Paulo, 1989.

GJERDE, B., SCHAEFFER, L. R. Body traits in rainbow trout. II Estimates of heritabilities and phenotypic and genetic correlations. Aquaculture. v. 80, p. $25-44,1989$.

GUNNES, K., GJEDREM, T. A genetic analysis of body weight and lenght in raibow trout reared in sea water for 18 months. Aquaculture. v. 24, p. 61-174, 1981.

LE CREN, E. D. The lenght-weight relationship and seasonal cycle in gonad weight and condition in the perch (Perca fluviatis). J. Anim. Ecol. v. 20, p. 201-219, 1951.

LEITE, R. G., VERANI, J. R., BASILE-MARTINS, M. A., GODINHO, H. M. FENERICH-VERANI, N. CESTAROLLI, M. A. Estudos biométricos do curimbatá, Prochilodus scrofa, em experimento de cultivo com suplementação alimentar. I. Morfometria. An. Simp. Bras. Aquic. III, São Carlos, p. 329-343, 1984.

MAINARDESPINTO, C.S.R., PAIVA, P. de, ANTONIUTTI, D. M., VERANI,
Tabela 7 - Taxa de crescimento médio (igr) conforme as idades e famílias de meio-irmãos maternos

\begin{tabular}{|c|c|c|c|}
\hline Fêmeas & Idade & Peso médio (g) & $\operatorname{Igr}(\%)$ \\
\hline 1 & $\begin{array}{c}60 \\
90 \\
150 \\
210 \\
270 \\
330\end{array}$ & $\begin{array}{c}1,18 \\
7,30 \\
21,98 \\
56,22 \\
111,25 \\
164,00\end{array}$ & $\begin{array}{l}- \\
6,07 \\
1,84 \\
1,56 \\
1,14 \\
0,65\end{array}$ \\
\hline 2 & $\begin{array}{c}60 \\
90 \\
150 \\
210 \\
270 \\
330\end{array}$ & $\begin{array}{c}1,61 \\
6,86 \\
18,42 \\
61,06 \\
110,09 \\
177,62\end{array}$ & $\begin{array}{l}- \\
4,83 \\
1,65 \\
2,00 \\
0,98 \\
0,80\end{array}$ \\
\hline 3 & $\begin{array}{c}60 \\
90 \\
150 \\
210 \\
270 \\
330\end{array}$ & $\begin{array}{c}1,20 \\
6,45 \\
16,76 \\
67,55 \\
127,87 \\
181,89\end{array}$ & $\begin{array}{c}- \\
5,60 \\
1,59 \\
2,32 \\
1,06 \\
0,59\end{array}$ \\
\hline 4 & $\begin{array}{c}60 \\
90 \\
150 \\
210 \\
270 \\
330\end{array}$ & $\begin{array}{c}1,99 \\
8,70 \\
14,23 \\
51,53 \\
95,62 \\
148,70\end{array}$ & $\begin{array}{l}- \\
4,90 \\
0,80 \\
2,10 \\
1,03 \\
0,74\end{array}$ \\
\hline 5 & $\begin{array}{c}60 \\
90 \\
150 \\
210 \\
270 \\
330\end{array}$ & $\begin{array}{c}1,24 \\
6,89 \\
20,56 \\
71,44 \\
107,43 \\
161,21\end{array}$ & $\begin{array}{c}- \\
5,70 \\
1,80 \\
2,10 \\
0,68 \\
0,68\end{array}$ \\
\hline
\end{tabular}

A taxa de crescimento médio (igr) indicou que o período em que ocorreu o maior crescimento foi em idades entre 60 e 90 dias para todas as famílias. Entre 90 e 150 dias houve um discreto crescimento devido ao tamanho dos peixes e o espaço disponível (tanque-rede). Entre 150 e 210 dias os animais apresentaram desenvolvimento acentuado, e nas idades de 270 a 330 dias o crescimento foi menos intenso.

J. R., JUSTO, C. L. Influência do arraçoamento no crescimento do curimbatá, Prochilodus scrofa, em tanques experimentais de cultivo. An. Simp. Bras. Aquic. III, São Carlos, p. 313-327, 1984.

REFSTIE, T.; STEINE, T. A. Selection experiments with salmon. III Genetic and environmental sources of variation in length and weight of atlhantic salmon in the fresh water phase. Aquaculture, v. 14, p. 221-234, 1978.

RICKER, W. E. Growth rates and models. In: HOAR, W.S.; RANDALL, D. J., BRETT, J.R. Fish physiology bioenergetics and growth. New York: Academic Press, 1979.

ROYCE, W. E. Introduction to the fishery science. New York: Academic Press, 1972.

SOKAL, R. R. \& ROHLF, F. J. Introduccion a la bioestatística. Barcelona: Editorial Reverte, 1980.

VERANI, J. R., MAINARDES PINTO, C. S. R., ANTONIUTTI, D. M.; STEMPNIEWSKI, H. L., PEDROSA, M. A. Crescimento do curimbatá Prochilodus scrofa, submetido a diferentes tipos de fertilização orgânica. Boletim Inst. Pesca, v. 16, p. 47-55, 1989.

WELCH, H, E., MILLS, K. H. Marking fish by scarring soft fin rays. Can. J. Fish. Aquat. Sci. v. 38, p. 1168-1170, 1981. 